\title{
HIGH-PRECISION RANGING BASED ON MULTISPECTRAL FULL-WAVEFORM LIDAR
}

\author{
B. Wang ${ }^{1}$, S. Song ${ }^{2, *}$, W. Gong ${ }^{1}$, S. Shi ${ }^{1}$, B. Chen ${ }^{1}$, J. Yang ${ }^{3}$, L. Du ${ }^{3}$, J. Sun ${ }^{3}$ \\ ${ }^{1}$ State Key Laboratory of Information Engineering in Surveying, Mapping and Remote Sensing, Wuhan University, Wuhan, China \\ - (bhwang810, weigong, shishuo, cbw_think)@whu.edu.cn \\ ${ }^{2}$ State Key Laboratory of Magnetic Resonance and Atomic and Molecular Physics, Wuhan Institute of Physics and Mathematics, \\ Chinese Academy of Sciences, Wuhan, China - songshalei@wipm.ac.cn \\ ${ }^{3}$ Faculty of Information Engineering, China University of Geosciences, Wuhan, China - (yangjian, dulin, sunjia)@cug.edu.cn
}

\section{Commission III, WG III/5}

KEY WORDS: B-spline, Full Waveform, Lidar Ranging, Multispectral Lidar, Parameters Extraction, Waveform Characteristic

\begin{abstract}
:
Full-waveform light detection and ranging (LiDAR) has become a well-established technique for remote sensing of surface topography and target characterization, offering numerous opportunities in investigation and interpretation of the structural diversity of surface coverage. However, most prevailing waveform decomposition methods employ the Gaussian function or some other probability distributions to model LiDAR waveforms of specific shapes, which cannot be universally used. Moreover, most of these waveform decomposition methods operate at a single laser wavelength and cannot be applied well to multispectral LiDAR (MSL) or hyperspectral LiDAR (HSL), which can simultaneously collect the spectral and geometric attributes with multiple transmitting laser wavelengths. In this paper, we propose a new multispectral B-spline waveform decomposition model to achieve high precision multi-target ranging. Considering both the spatial consistency of each channel and the irregular shape of the received waveform, LiDAR waveforms are modelled by B-splines rather than any other specific probability distribution. Thus, the proposed method can be extended to other FWMSL data benefiting from the flexibility of B-splines on fitting arbitrary curves. Both simulated MSL echoes and a measured dataset from a FWMSL system with three wavelengths of 556, 670, and $780 \mathrm{~nm}$ were used in this study. Compared with two single wavelength waveform decomposition models and a multispectral waveform decomposition model based on Gaussian function, the proposed method has excellent robustness for processing different shapes of waveforms and can improve ranging accuracy significantly for irregular waveforms.
\end{abstract}

\section{INTRODUCTION}

Full-waveform light detection and ranging (LiDAR) has become a well-established technique for remote sensing of surface topography and target characterization (Sumnall, Hill and Hinsley 2016, Li et al. 2016), offering numerous opportunities in investigation and interpretation of the structural diversity of surface coverage. In particular, multiple targets along the laser path can be detected through the waveform decomposition of the full waveform data, thereby obtaining ranging information by calculating the time of flight between the transmitted pulse and each waveform component.

However, most prevailing waveform decomposition methods employ the Gaussian function (Hofton, Minster and Blair 2000, Wagner et al. 2006) or some other probability distributions such as lognormal distribution, generalized normal distribution, and Burr distribution to model LiDAR waveforms of specific shapes (Chauve et al. 2009), which cannot be universally used. Moreover, most of these waveform decomposition methods operate at a single laser wavelength and cannot be applied well to multispectral LiDAR (MSL) or hyperspectral LiDAR (HSL). MSL and HSL have been proposed to simultaneously collect the spectral and geometric attributes with multiple transmitting laser wavelengths in recent years, widely adopted in vegetation detection and land cover mapping (Wei et al. 2012, GuerreroRascado et al. 2014, Song et al. 2019, Hakala et al. 2012, Scaioni et al. 2018).

In this paper, we propose a new multispectral B-spline waveform decomposition model to achieve high precision multi-target ranging. The proposed method takes into account the spatial consistency of each channel in order to explore the potential advantages of the full waveform multispectral LiDAR (FWMSL) system. This is beneficial to improve the multitarget detection performance of channels with lower signal-tonoise ratios (SNR) with the help of channels with high SNR. Moreover, LiDAR waveforms are modelled by B-splines rather than any other specific probability distribution, and the shape of the received waveform is considered as a mixture of finite transmit pulses after translation and scaling transformations. Thus, the proposed model can be extended to other FWMSL data benefiting from the flexibility of B-splines on fitting arbitrary curves.

\footnotetext{
* Corresponding author
} 


\section{MATERIALS AND METHODS}

\subsection{FWMSL system}

The FWMSL system can record both the transmitted pulse and receiving echo with wavelengths of 556,670 , and $780 \mathrm{~nm}$ The waveforms of the three receiving channels are digitized at a sampling rate of $1.8 \mathrm{GHz}$ in a 12-bit analog-to-digital converter (SP Devices, ADQ412). The solid-state lasers at these three wavelengths are synthesized into a beam at a repetition rate of $20 \mathrm{kHz}$ with average power of $100 \mathrm{~mW}$ for each laser. The divergence angle of each beam is approximately $0.6 \mathrm{mrad}$, providing a laser footprint of approximately $3 \mathrm{~cm}$ in diameter at a detection distance of $50 \mathrm{~m}$ The pulse widths of these lasers were $2 \mathrm{~ns}$.

\subsection{B-spline-based modelling}

On the basis of prevailing methods, the received LiDAR waveform echo can be regarded as the superposition of finite echo components and background noise (Mallet and Bretar 2009).

$$
f(x ; \theta)=\sum_{m=1}^{M} f\left(x ; \theta_{m}\right)+\text { noise }
$$

where $\mathrm{M}$ is the number of the components of the echo waveform, $\theta_{m}$ represents the descriptive parameters of the $m$ th components, and noise refers to the mean noise level, which is usually estimated by averaging the amplitudes of the first or the last few waveform samples and is then removed from the raw waveform signals (Hofton et al. 2000).

Generally, assuming that the laser pulses are all Gaussian, several symmetric Gaussian functions are used to fit the full waveform echo, each function corresponding to an echo component (Hofton et al. 2000, Wagner et al. 2006, Chauve et al. 2008, Lin, Mills and Smith-Voysey 2010).

$$
f^{G}\left(x ; \theta_{m}\right)=a_{m} \exp \left(-\frac{\left(x-\mu_{m}\right)^{2}}{2 \sigma_{m}^{2}}\right)
$$

where $f^{G}\left(x ; \theta_{m}\right)$ refers to the Gaussian model, and $a_{m}, \mu_{m}$, and $\sigma_{m}$ represent the amplitude, peak position, and standard deviation of the $m$ th Gaussian component, respectively.

Since the assumption of the Gaussian shape is sometimes oversimplified, especially for trailing waveforms, the Gaussian decomposition method cannot always perform well on real LiDAR waveforms. Therefore, the B-spline technique can be used to fit pulses of any shape and simplify the calculation of echo parameters, including echo width, amplitude, and center position, where the center position is a key parameter for ranging. The B-spline method has been widely used in curve fitting applications (Gruen and Akca 2005, Guan et al. 2014, Shen et al. 2017). Its formula is given by

$$
B(x)=\sum_{i=1}^{I} b_{i} B_{i}(x)
$$

where $b_{i}$ is the control point, $P$ is the degree of the spline, and $B_{i}(x)$ is the basis function of the B-spline and is given by a recursion formula (Roncat, Bergauer and Pfeifer 2011). The parameter describing $B_{i}(x)$ is called knot $u_{i}$.

Assuming that the echo shape of the received waveform can be approximated by the corresponding transmit pulse through linear translation and scaling, the knots and control points of the corresponding two B-spline curves should actually meet

$$
\begin{aligned}
& \mathbf{u}_{m}=\lambda_{m} \mathbf{u}_{t w}+\mu_{m} \\
& \mathbf{b}_{m}=\kappa_{m} \mathbf{b}_{t w}
\end{aligned}
$$

where $\mathbf{u}_{m}$ and $\mathbf{b}_{m}$ are the knots and control points of $m$ th Bspline component, respectively. $\mathbf{u}_{t w}$ and $\mathbf{b}_{t w}$ are the knots and control points of the transmit pulse, respectively. $\lambda_{m}$ and $\mu_{m}$ are the scale and translation coefficients in the range direction, respectively, and $\kappa_{m}$ refers to the scale factor in the amplitudes, corresponding to $m$ th B-spline component.

\subsection{Multispectral waveform decomposition}

Similar to the Gaussian decomposition method, the peak and inflection points can be combined in the preprocessing stage to estimate the number of echo components and their initial parameters (Song et al. 2019, Wang et al. 2015). The translation factor $\mu_{m}$ in the range direction can be initialized to $\left(P_{m}^{0}-P_{t w}\right)$, where $P_{m}^{0}$ is the rough position of the $m$ th component, and $P_{t w}$ is the peak position of the transmitted waveform.

The pulse width of the $m$ th echo is initialized by that of the transmitted waveform assuming that the echo is not widened due to the tilt or roughness of the target. Therefore, the initial scale factor $\lambda_{m}^{0}$ in the range is given by 1 . The scale factor of the amplitudes is initialized as $\frac{A_{m}^{0}}{A_{t w}}$, where $A_{m}^{0}$ and $A_{t w}$ are the maximal amplitudes of the $m$ th component and the transmitted waveform, respectively.

The Levenberg-Marquardt optimization (Wagner et al. 2006, Mountrakis and Li 2017) is then carried out on the initially estimated components at each receiving channel using the Bspline model in order to obtain closer component parameters that are closer to the true values. During the parameter optimization procedure, potential echo components are added ordered by energy level. Thereby, the model parameters $\lambda_{m}$, $\kappa_{m}$, and $\mu_{m}$ are accurately acquired.

After retrieving the accurate model parameters, the waveform properties can be then calculated. From Eq. (4), the position $P_{m}^{B}$, width $W_{m}^{B}$ and amplitude $A_{m}^{B}$ of the $m$ th B-spline component can be derived.

$$
\begin{aligned}
& P_{m}^{B}=\lambda_{m} P_{t w}+\mu_{m} \\
& W_{m}^{B}=\lambda_{m} W_{t w} \\
& A_{m}^{B}=\kappa_{m} A_{t w}
\end{aligned}
$$


At this point, the waveform component of each receiving channel can be obtained. However, the waveform component decomposition results of the three receiving channels may not be uniform, which is inconsistent with the real scene. In theory, the echo components of each channel should have the same position distribution. Therefore, the waveform components of each channel need to be further judged whether they correspond to the same target, so as to obtain more accurate waveform parameters.

The components at different wavelengths correspond to the same target if the difference between the position of these components is smaller than one sixth of the width of the transmitted pulse. In this way, the position average and width average of these Gaussian components are taken as the initial parameters of the target. Otherwise, these components at multiple wavelengths are from two or three different targets. The position and width at each wavelength are all taken as the initial values. Thus, a new set of echo properties is generated as $\left(P_{m}^{B}, W_{m, j}^{B}, A_{m, j}^{B}\right)$, where $j$ is the $j$ th channel. The distance of targets can be calculated by the time of flight between the transmitted pulse and each waveform component, which is related to the position of echo components. Thus, the distance of the $m$ th target can be calculated as

$$
\text { distance }_{m}=\left(P_{m}^{B}-P_{t w}\right) / 2
$$

\subsection{Data description}

To evaluate the efficiency and feasibility of the proposed method, two datasets were utilised: a synthetic dataset and a measured dataset from the FWMSL system. The simulated waveforms were designed to mimic the real echo waveforms with different levels of skewness and kurtosis. Noise was introduced with an absolute standard deviation value of 0.5 . The sampling rate was set to $1.8 \mathrm{G}$ samples per second.

As for the measured dataset, two standard white boards with 99\% reflectivity at wavelengths between 200 and $2500 \mathrm{~nm}$ were detected. In the experiment, the laser spot partially hit both two white boards. We collected the superposition of the laser pulses scattered from these two planes. The distance between the two targets was changed by moving the second plane away from the first plane from $5 \mathrm{~m}$ to $20 \mathrm{~m}$ in steps of 1 m.

\subsection{Validation}

The proposed model, referred to as MB, was compared with two single wavelength waveform decomposition models, i.e. Gaussian function (SG) (Hofton et al. 2000) and B-splinebased model (SB) (Shen et al. 2017), and the multispectral waveform decomposition model based on Gaussian function (MG) (Song et al. 2019). Two statistical estimators have been commonly employed to evaluate the ranging performance, namely precision and accuracy. The former represents systematic random errors, while the latter represents systematic errors. where $E_{\text {pre }}$ is the ranging precision measured in a certain ranging position, $\mathrm{N}$ is the sampled number. $R_{i}$ denotes the $i$ th measured data and $\bar{R}$ is the average of the measured data.

$$
E_{\text {acu }}=\frac{1}{N} \sum_{i=1}^{N}\left(R_{i}-R_{\text {true }}\right)
$$

where $E_{a c u}$ is the ranging accuracy measured in a certain ranging position, the actual value $R_{\text {true }}$ is obtained from the reference ranging finder.

\section{RESULTS AND DISCUSSION}

In order to evaluate the running efficiency of the four methods, i.e. $\mathrm{MB}, \mathrm{SG}, \mathrm{SB}$, and $\mathrm{MG}$, the execution time of ranging based on each method is calculated based on CPU processing. All the programs are running on a computer with $4 \mathrm{~GB}$ (gigabyte) of RAM (random-access memory), and an Intel(R) Core(TM) i56500U CPU @ $3.20 \mathrm{GHz}$. The average execution time of the MB, SG, SB, and MG are calculated to be 316.2, 621.4, 567.4, and $632.3 \mathrm{~ns}$ for each laser shot with three receiving channels, respectively. This result indicates that the proposed method runs more efficiently than the other three methods because of simpler mathematic expression and less iterations.

Moreover, in order to demonstrate the influence of different waveform decomposition, the results of $\mathrm{MB}, \mathrm{SG}, \mathrm{SB}$, and $\mathrm{MG}$ are compared with each other given the same input signal. The ranging precision and accuracy estimated with the four methods for the simulated and measured waveforms are shown in Figure 1. Firstly, the ranging precision of all waveform decomposition methods decrease with the increase of the distance, among the four methods the proposed method has the best precision. Secondly, the ranging accuracy tends to be stable with increasing distance.

The simulated waveforms contain echoes with different shape characteristics, including symmetrical waveforms similar to Gaussian, trailing echoes, and other arbitrary shape echoes. Figure 1 (a) and (b) demonstrate the results of simulated data. The results show that the proposed method based on the multispectral B-spline model has better ranging performance than the other three methods. The ranging accuracy of the Gaussian based method ( $\mathrm{SG}$ and $\mathrm{MG}$ ) is not satisfied and much worse than that of SB and MB when the waveform is sharply-peaked or heavy-tailed. Benefiting from the abundant spectral information with multiple wavelengths, the waveform components decomposed based on the MB model exhibit high consistency at each wavelength and high ranging precision, which is more reliable and offers considerable improvement over the SB model.

$$
E_{p r e}=\sqrt{\frac{1}{N} \sum_{i=1}^{N}\left(R_{i}-\bar{R}\right)^{2}}
$$



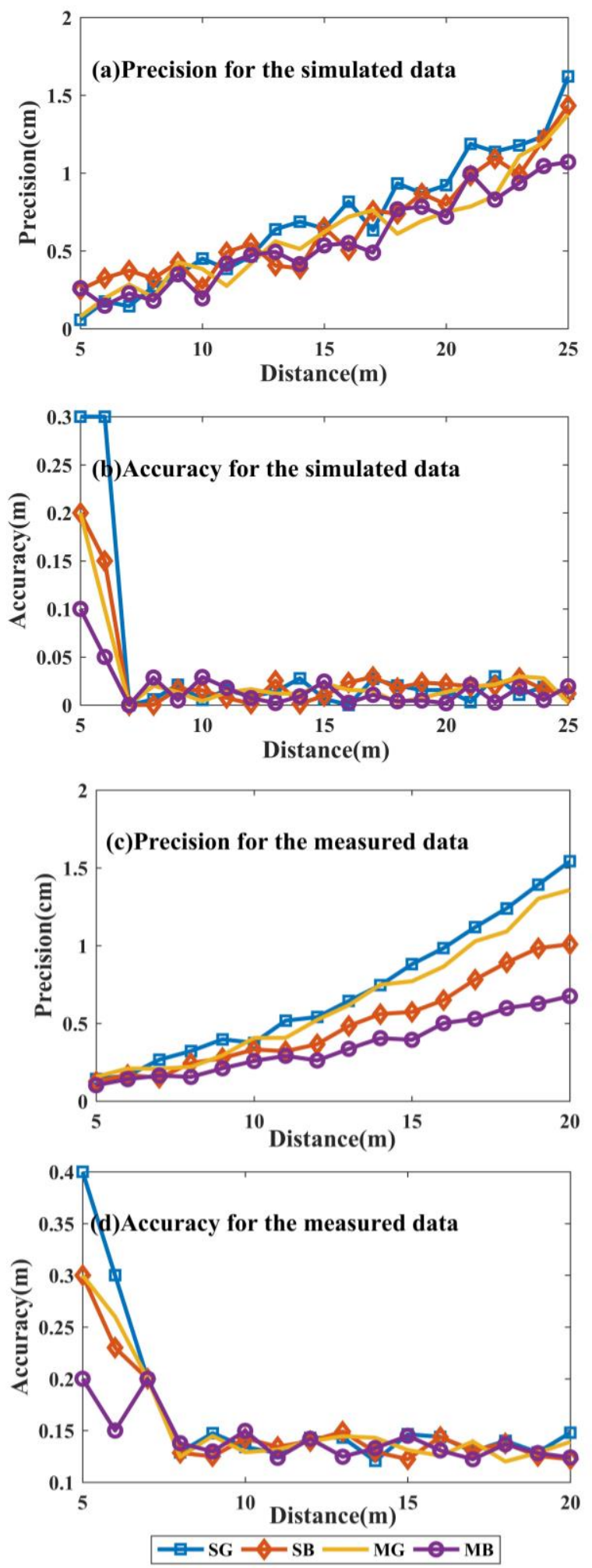

Figure 1. Precision and accuracy comparison of four methods for the simulated and measured waveforms. SG, SB, MG, and MB stand for Gaussian function, B-spline-based model, the multispectral waveform decomposition model based on Gaussian function, and the proposed method.

Additionally, Figure 1 (c) and (d) displays the comparison results for the measured data. From the definition in Section 2.5, two indicators including bias and nonlinearity are calculated to evaluate the ranging accuracy. The results show that the bias and nonlinearity of the proposed method is less than the other three methods. In order to give a specification of the four methods, their precision is given the maximum precision value over the entire measurement range. The specification is present in Table 1.

\begin{tabular}{|c|c|c|}
\hline Methods & Maximum precision $(\mathrm{cm})$ & Mean precision $(\mathrm{cm})$ \\
\hline SG & 1.51 & 0.72 \\
SB & 1.00 & 0.49 \\
MG & 1.42 & 0.64 \\
MB & 0.74 & 0.34 \\
\hline
\end{tabular}

Table 1 . The ranging accuracy and precision of measured waveforms

Since the waveform measured by the FWMSL system is slightly tailed, the B-spline model seems to be more suitable to fitting the transmitted pulse than Gaussian function. Moreover, the multi-target ranging information retrieved based on SG varied at each wavelength, inconsistent with the actual position distribution of targets while the waveform components exhibit a high consistency at each wavelength when using MG (Song et al. 2019). Similarly, the proposed method makes full use of the advantages of FWMSL, with the help of the component position distribution of multiple channels to obtain more accurate waveform information. This study aims to achieve multi-target high-precision ranging using FWMSL data by improving the waveform decomposition method. The accuracy of the proposed method may be further improved by calibration or improving the detection and data acquisition modules.

\section{CONCLUSIONS}

In conclusion, this paper proposed a new multispectral Bspline waveform decomposition model to achieve high precision multi-target ranging. The ranging performance of the proposed method was evaluated using both simulated and measured data, compared with the typical Gaussian function involving single/multiple wavelengths and the basic B-spline model based on a single wavelength. The results show that the proposed method has excellent robustness for processing different shapes of waveforms, due to the flexibility of B-spline fitting arbitrary curves. The proposed method takes advantage of multispectral full-waveform data to improve ranging accuracy significantly. Furthermore, the proposed method can be extended to other full waveform MSL or HSL systems of arbitrary waveforms.

\section{ACKNOWLEDGEMENTS}

This work was supported by the National Key R\&D Program of China (2018YFB0504500) and NSFC (41571370).

\section{REFERENCES}

Chauve, A., C. Mallet, F. Bretar, S. Durrieu, M. P. Deseilligny \& W. Puech. 2008. Processing full-waveform lidar data: modelling raw signals. In International archives of photogrammetry, remote sensing and spatial information sciences 2007, 102-107.

Chauve, A., C. Vega, S. Durrieu, F. Bretar, T. Allouis, M. Pierrot Deseilligny \& W. Puech (2009) Advanced fullwaveform lidar data echo detection: Assessing quality of 
derived terrain and tree height models in an alpine coniferous forest. International Journal of Remote Sensing, 30, 5211-5228.

Gruen, A. \& D. Akca (2005) Least squares 3D surface and curve matching. ISPRS Journal of Photogrammetry and Remote Sensing, 59, 151-174.

Guan, H., J. Li, Y. Yu, C. Wang, M. Chapman \& B. Yang (2014) Using mobile laser scanning data for automated extraction of road markings. ISPRS Journal of Photogrammetry and Remote Sensing, 87, 93-107.

Guerrero-Rascado, J. L., R. Facundes da Costa, A. E. Bedoya, R. Guardani, L. Alados-Arboledas, A. E. Bastidas \& E. Landulfo (2014) Multispectral elastic scanning lidar for industrial flare research: characterizing the electronic subsystem and application. Opt Express, 22, 31063-77.

Hakala, T., J. Suomalainen, S. Kaasalainen \& Y. Chen (2012) Full waveform hyperspectral LiDAR for terrestrial laser scanning. Optics Express, 20, 7119.

Hofton, M. A., J. B. Minster \& J. B. Blair (2000) Decomposition of laser altimeter waveforms. IEEE Transactions on Geoscience \& Remote Sensing, 38, 1989-1996.

Li, W., Z. Niu, J. Li, H. Chen, S. Gao, M. Wu \& D. Li (2016) Generating pseudo large footprint waveforms from small footprint full-waveform airborne LiDAR data for the layered retrieval of LAI in orchards. Opt Express, 24, 10142-56.

Lin, Y.-C., J. P. Mills \& S. Smith-Voysey (2010) Rigorous pulse detection from full-waveform airborne laser scanning data. International Journal of Remote Sensing, 31, 1303-1324.

Mallet, C. \& F. Bretar (2009) Full-waveform topographic lidar: State-of-the-art. ISPRS Journal of Photogrammetry and Remote Sensing, 64, 1-16.

Mountrakis, G. \& Y. Li (2017) A linearly approximated iterative Gaussian decomposition method for waveform LiDAR processing. ISPRS Journal of Photogrammetry and Remote Sensing, 129, 200-211.
Roncat, A., G. Bergauer \& N. Pfeifer (2011) -spline deconvolution for differential target cross-section determination in full-waveform laser scanning data. ISPRS Journal of Photogrammetry and Remote Sensing, 66, 418-428.

Scaioni, M., B. Höfle, A. P. Baungarten Kersting, L. Barazzetti, M. Previtali \& D. Wujanz. 2018. Methods from information extraction from lidar intensity data and multispectral lidar technology. In ISPRS Technical Commission III on Remote Sensing.

Shen, X., Q.-Q. Li, G. Wu \& J. Zhu (2017) Decomposition of LiDAR waveforms by B-spline-based modeling. ISPRS Journal of Photogrammetry and Remote Sensing, 128, 182-191.

Song, S., B. Wang, W. Gong, Z. Chen, X. Lin, J. Sun \& S. Shi (2019) A new waveform decomposition method for multispectral LiDAR. ISPRS Journal of Photogrammetry and Remote Sensing, 149, 40-49.

Sumnall, M. J., R. A. Hill \& S. A. Hinsley (2016) Comparison of small-footprint discrete return and full waveform airborne lidar data for estimating multiple forest variables. Remote Sensing of Environment, 173, 214-223.

Wagner, W., A. Ullrich, V. Ducic, T. Melzer \& N. Studnicka (2006) Gaussian decomposition and calibration of a novel small-footprint full-waveform digitising airborne laser scanner. ISPRS Journal of Photogrammetry and Remote Sensing, 60, 100-112.

Wang, C., Q. Li, Y. Liu, G. Wu, P. Liu \& X. Ding (2015) A comparison of waveform processing algorithms for singlewavelength LiDAR bathymetry. ISPRS Journal of Photogrammetry and Remote Sensing, 101, 22-35.

Wei, G., S. Shalei, Z. Bo, S. Shuo, L. Faquan \& C. Xuewu (2012) Multi-wavelength canopy LiDAR for remote sensing of vegetation: Design and system performance. ISPRS Journal of Photogrammetry and Remote Sensing, 69, 1-9. 